\title{
Prevalence of Complementary And Alternative Medicine Use In Brazil: Results of The National Health Survey, 2019
}

Patricia de Moraes Mello Boccolini ( $\sim$ patriciaboccolini@yahoo.com.br)

Faculdade de Medicina de Petrópolis (FMP/UNIFASE)

Karine de Lima Sírio Boclin

Universidade Estácio de Sá, Rio de Janeiro

Islândia Maria Carvalho de Sousa

Fundação Oswaldo Cruz, Instituto de Pesquisas Aggeu Magalhães

Cristiano Siqueira Boccolini

Fundação Oswaldo Cruz

\section{Research Article}

Keywords: complementary and alternative medicine, prevalence, population survey.

Posted Date: December 1st, 2021

DOI: https://doi.org/10.21203/rs.3.rs-1054236/v1

License: (c) (i) This work is licensed under a Creative Commons Attribution 4.0 International License. Read Full License 


\section{Abstract}

Background: In recent decades, it has been possible to observe an increase in Complementary and Alternative Medicine (CAM) usage globally for both disease prevention and health promotion purposes. We aim to analyze factors associated with the prevalence of CAM use in Brazil.

Methods: Observational study with data from the 2019 National Health Survey that evaluated a sample of Brazilian adults. The outcome was CAM use, such as acupuncture, homeopathy, medicinal plants and herbal medicines, meditation, and yoga in the last 12 months. A logistic regression model with a $95 \%$ confidence interval was estimated to assess factors associated with CAM use.

Results: The prevalence of CAM use in 2019 was $5.2 \%$ (CI99\%=4.8\%-5.6\%), the most used being: medicinal plants and herbal medicines, with a prevalence of $3.0 \%(\mathrm{Cl} 99 \%=2.7-3.33)$ followed by: acupuncture 1.4\% (Cl99\%=1.3-1.6) homeopathy 0.9\% (Cl99\%=0.7-1.0), meditation 0.7\% (Cl99\%=0.6-0.8) and yoga $0.4 \%$ (CI99\%=0.4-0.5). We observed important differences in CAM use in Brazil, with a higher prevalence in the North Region, 3.7\% (CI99\%=2.81-4.75), where herbal medicines were more frequent the in the other regions. After estimating an adjusted model, women, older people, and people with a higher level of education and per capita income were the ones who most used all types of CAM. The practice of yoga stands out among women 3.6\% (CI99\%=2.49-5.28) and among individuals with higher per capita income 7.5\% (Cl99\%=2.97-18.93); meditation among individuals with higher educational level $13.4 \%$ $(\mathrm{Cl} 99 \%=6.41-28.33)$ and acupuncture for those who declared regular or poor health $1.9 \%(\mathrm{Cl} 99 \%=1.51$ 2.39).

Conclusions: We recommend that the Ministry of Health expand CAM access to Unified Health System users and promote health professionals' conscious and guided use for the Brazilian population.

\section{Background}

In recent decades, it has been possible to observe an increase in the use of the so-called Traditional, Complementary and Integrative Medicine (TCIM) in the world for both disease prevention and health promotion purposes $^{1-4}$.

Limitations of the biomedical model towards the health challenges due to the epidemiological and demographic transition can partly explain this increase. Along with this, the World Health Organization (WHO) stimulates countries to implement these practices in their health systems ${ }^{4,5}$.

The expression Traditional, Complementary and Integrative Medicine, coined by the WHO, is known locally in Brazil as Integrative and Complementary Practices in Health and was implemented in the Unified Health System (SUS) ${ }^{5}$. We will adopt the term Complementary and Alternative Medicine (CAM) in this study regarding these terms. 
It is essential to state that indigenous and traditional medicines are not enrolled in the Brazilian CAM definition. The main reason for this is that indigenous public health care in Brazil has a specific primary care model for indigenous populations through multidisciplinary health teams and is organized together with indigenous authorities as a subsystem ${ }^{6}$.

Since 2006, Brazil has had a National Policy on Integrative and Complementary Practices in Health with 29 practices currently recognized by the Ministry of Health of Brazil: homeopathy; Traditional Chinese Medicine; anthroposophical medicine; social thermalism/crenotherapy; medicinal plants and herbal medicine; art therapy, Ayurveda, biodance, laying on of hands, circular dance, meditation, music therapy, naturopathy, osteopathy, chiropractic, reflexology, Shantala, integrative community therapy, family constellation, chromotherapy, aromatherapy, geotherapy, bioenergetics, apitherapy, hypnotherapy, reiki, therapy floral, ozone therapy and yoga ${ }^{7,8}$. CAM in Brazil originates from complex systems such as Acupuncture, Homeopathy, Ayurveda and can be organized according to treatment methods, which in this case are: herbal medicine and medicinal plants, manual care (acupuncture, chiropractic, osteopathy, massage), body-mind therapies (tai chi chuan, yoga, lian gong, meditation, bioenergetics) or support group therapy such as the Brazilian methodology known as integrative community therapy ${ }^{6}$.

Worldwide, the prevalence of CAM use in the general population can range from $10-75 \%{ }^{9}$. A Brazilian study with data from the PNS-2013 (Brazilian National Health Survey) observed that more than seven million adults reported using CAM, representing a prevalence of $4.5 \%{ }^{10}$. When comparing NHS data with those from other countries, we can observe variability in prevalence estimates: in the United States, the prevalence of CAM use was $33 \%$, in Germany, it was $40 \%$, and in Malaysia, it was $56 \%^{11-13}$. Thus, this article aims to analyze the prevalence of CAM use in the Brazilian adult population.

\section{Methods}

\section{Study design, objective, and sampling}

This study is a population epidemiological survey with a representative sample of the Brazilian adult population residing in permanent households in Brazil, the 2019 National Health Survey (PNS-2019), whose secondary data were obtained from https://www.ibge.gov.br/estatisticas/sociais/saude/9160pesquisa-nacional-de-saude.html?=\&t=microdados ${ }^{14}$.

The PNS-2019 sample consisted of clusters in three stages, with stratification of census tracts (primary sampling units - PSU), where, first, the UPA were randomly selected, followed by random selection of a fixed number of permanent households, and, finally, a resident over 15 years of age or older was randomly selected in that household from a list of eligible residents. The selected resident answered an individual questionnaire, and two additional questionnaires, one household and the other for all residents were answered by the selected subject or by a family member who could provide detailed information on socioeconomic and health. Further details can be found at https://www.pns.icict.fiocruz.br/delineamentoda-pns/. 
Since this is a sample with a complex design, expansion factors were calculated, including correction factors for losses, followed by weighing and calibration based on population projections. The PNS-2019 calibration process allowed for comparability with the PNS-2013. The complex sample design was incorporated into all estimates in this study.

\section{Outcome And Associated Variables}

For this study, the outcome was the use of one or more Complementary and Alternative Medicine (CAM), which was obtained through the question: "In the last twelve months, used treatments such as acupuncture, homeopathy, medicinal plants and herbal medicine, meditation, yoga, Tai chi chuan, Lian gong or any other integrative and complementary health practice?". For the subjects who answered positively to this question, additional questions were asked to detail which CAM was (were) used, namely: Acupuncture, Homeopathy, Medicinal Plants, Meditation, Yoga, Tai chi chuan (or Lian gong, or Qi gong), Community Therapy, or others, multiple responses are possible. The answers were considered individually and later computed, identifying how many CAM listed each subject used.

We assessed the access to healthcare using the question: "When did _ last see a doctor?" The answer options were: "Up to one year" (we considered yes, had access to health), "more than one year to 2 years" (we considered as no, had no access to health), "more than two years to 3 years" (we considered as no, did not have access to health), "more than three years" (we considered as no, did not have access to health), and "never went to the doctor" (we considered as no, did not have access to health).

The per capita income variable was calculated and converted to the Brazilian minimum wage. The minimum wage in Brazil in 2019 was $R \$ 998.00$. This amount was divided by the monthly average of the dollar in that same year (\$3.946). We then categorized into up to $\$ 126.46$ (equivalent to up to $1 / 2$ minimum wages -MW), over $\$ 136.46$ and up to $\$ 252.91$ (over $1 / 2 \mathrm{MW}$ and up to $1 \mathrm{MW}$ ) - Over $\$ 252.91$ and up to $\$ 502.8$ (More than $1 \mathrm{MW}$ and up to $2 \mathrm{MW}$ ) - More than $\$ 502.83$ (More than $2 \mathrm{MW}$ ).

The covariates used in this study were the Brazilian regions in which the respondent lives, sex (male, female), age group (18-39, 40-59, 60 or older), educational level (complete elementary school, complete high school, university degree or more), per capita income (up to $1 / 2$, more than $1 / 2$ and up to 1 , more than one up to 2, and more than two minimum wages $-\mathrm{MW}$ ), color/race (non-white and white), self-assessment in health (good/very good, fair/bad/very bad), use of private health insurance (no, yes), and access to health care in the last 12 months (no, yes).

Initially, we estimated the prevalence of CAM use (one or more CAMs) by categories of sociodemographic characteristics, self-assessment in health, and access to health services. Then, we estimated the association between exposure covariates and the primary outcome (use of one or more CAMs) employing a generalized linear model with a logistic link function and a $99 \%$ confidence interval. 
In the next step, the prevalence and confidence intervals of $99 \%$ of the use of the four most frequently used CAMs (Acupuncture, Homeopathy, Medicinal Plants, Meditation, Yoga) were estimated, according to the categories of the covariates or exposure variables, with a confidence interval $99 \%$.

The least used CAMs, such as Integrative and Community Therapy, Tai chi or Lian gong, or Qi gong and Auriculotherapy, were not tabulated.

Then, generalized linear models were estimated, with logistic link function, $99 \%$ confidence interval, and $1 \%$ alpha, with one model estimated for each of the four most used CAMs and another model for those who used one or more CAMs. All covariates or exposure variables were considered concomitantly in these models, regardless of their statistical significance level.

In all stages, we considered the sample weights and the complex design of the sample. All analyses were conducted using the $\mathrm{R}$ version 3.6.0 program.

Finally, to compare the prevalence of CAM use in 2019, we estimated CAM prevalence data from the 2013 National Health Survey (PNS-2013), incorporating the complex sample design and 95\% confidence interval. In the PNS-2013 edition, the participants reported only acupuncture, homeopathy, and medicinal and herbal plants (further details in Boccolini \& Boccolini, 2020), limiting the comparison with the PNS 2019 edition. CAM utilization data from PNS-2013 were compared with those from PNS-2019, considering differences in point prevalence and the absence of interpolation of the $95 \%$ confidence intervals.

\section{Results}

The PNS-2019 visited 108,525 households (with a non-response rate of $8.1 \%$ ), and 94,114 interviews were carried out (with a non-response rate of $6.4 \%$ ).

The prevalence of CAM use in Brazil in 2019 was 5.2\% (CI99\%=4.8\%-5.6\%), being higher in the North and South macro-regions, among female individuals, with higher education, higher income, in older age groups and of white color/race. Regarding health characteristics, the prevalence was higher among individuals who reported a worse health situation, had a private health plan, and had greater healthcare access (Table 1). 
Table 1

Prevalence of Complementary and Alternative Medicine (CAM) use, according to sociodemographic characteristics, (Brazil, PNS, 2019) e.

\begin{tabular}{|c|c|c|}
\hline Variables & Prevalence (\%) a & $99 \% \mathrm{Cl}^{b}$ \\
\hline \multicolumn{3}{|l|}{ Region } \\
\hline Southeast & 5.0 & $4.4-5.6$ \\
\hline South & 6.1 & $5.3-6.8$ \\
\hline Midwest & 4.2 & $3.5-5.0$ \\
\hline Northeast & 4.9 & $4.1-5.7$ \\
\hline North & 6.6 & $5.7-7.9$ \\
\hline \multicolumn{3}{|l|}{ Sex } \\
\hline Male & 3.8 & $3.5-4.2$ \\
\hline Female & 6.4 & $6.0-6.9$ \\
\hline \multicolumn{3}{|l|}{ Age Group } \\
\hline 18 to 39 years old & 4.1 & $3.7-4.5$ \\
\hline 40 to 59 years old & 5.8 & $5.3-6.3$ \\
\hline 60 years old or more & 6.5 & $5.9-7.1$ \\
\hline \multicolumn{3}{|l|}{ Educational level ${ }^{\mathrm{c}}$} \\
\hline Complete Elementary school & 4.2 & $3.7-4.6$ \\
\hline Complete High school & 3.7 & $3.3-4.1$ \\
\hline University degree or more & 9.9 & $9.0-10.8$ \\
\hline \multicolumn{3}{|l|}{ Per capita income ${ }^{d}$} \\
\hline Up to $1 / 2 \mathrm{MW}$ & 3.7 & $3.1-4.3$ \\
\hline Over $1 / 2 \mathrm{MW}$ and up to $1 \mathrm{MW}$ & 3.5 & $3.1-4.0$ \\
\hline Over $1 \mathrm{MW}$ and up to $2 \mathrm{MW}$ & 4.4 & $3.9-5.0$ \\
\hline Over $2 \mathrm{MW}$ & 10.1 & $9.1-11.0$ \\
\hline \multicolumn{3}{|l|}{ Race } \\
\hline Non-white & 4.4 & $4.0-4.8$ \\
\hline White & 6.2 & $5.7-6.8$ \\
\hline
\end{tabular}




\begin{tabular}{|c|c|c|}
\hline Variables & Prevalence (\%) a & $99 \% \mathrm{Cl}^{\mathrm{b}}$ \\
\hline Good/very good & 4.7 & $4.4-5.1$ \\
\hline Fair/bad/very bad & 6.2 & $5.6-6.7$ \\
\hline \multicolumn{3}{|c|}{ Private Health Insurance } \\
\hline No & 3.9 & $3.5-4.3$ \\
\hline Yes & 8.7 & $7.9-9.5$ \\
\hline \multicolumn{3}{|c|}{ Access to health services } \\
\hline No & 2.9 & $2.5-3.3$ \\
\hline Yes & 5.9 & $5.5-6.3$ \\
\hline Brazil (total) & 5.2 & $4.8-5.6$ \\
\hline \multicolumn{3}{|c|}{ a Prevalence considering the complex sample design } \\
\hline \multicolumn{3}{|c|}{ b $99 \%$ confidence interval considering the complex sample design } \\
\hline \multirow{2}{*}{\multicolumn{3}{|c|}{$\begin{array}{l}\text { c } \text { The variable education categories "illiterate and incomplete elementary school" were aggregated. } \\
\text { d Per capita income: Up to } \$ 126.46 \text { (equivalent to Up to } 1 / 2 \text { Minimum Wage -MW), over } \$ 136.46 \text { and up } \\
\text { to } \$ 252.91 \text { (over } 1 / 2 \mathrm{MW} \text { and up to } 1 \mathrm{MW} \text { ) - Over } \$ 252.91 \text { and up to } \$ 502.8 \text { (More than } 1 \mathrm{MW} \text { and up to } \\
2 \mathrm{MW} \text { - Over } \$ 502.83 \text { (More than } 2 \mathrm{MW} \text { ). }\end{array}$}} \\
\hline & & \\
\hline \multicolumn{3}{|c|}{$\begin{array}{l}\text { e Sample of individuals aged } 18 \text { years and older who answered the } 2019 \text { National Health Survey } \\
\text { individual questionnaire }\end{array}$} \\
\hline
\end{tabular}

In adjusted analyses, individuals from the South, Northeast, and North regions were more likely to report the CAM use when compared to individuals from the Southeast region. Higher chances of CAM use were also found among women, with complete higher education, per capita family income greater than two minimum wages, and aged 40 years or more when compared to the reference categories (male sex, complete elementary school, up to half the minimum wage and between 18 and 39 years of age). Reporting worse health status, having a private health insurance plan, and access to healthcare are also associated with greater CAM use (Table 2).

Table 2. Factors associated with the use of Complementary and Alternative Medicine, (Brazil, PNS,2019). 
Prevalence of CAM use ${ }^{a}(99 \% \mathrm{Cl})^{b}$ by sociodemographic variables

$\mathrm{AOR}(\%)^{\mathrm{c}} \quad 99 \% \mathrm{Cl}$

\section{Region}

Southeast

South

Midwest

1.00

1.26

$1.05-1.52$

Northeast

0.92

$0.74-1.15$

Northeast

1.32

$1.09-1.61$

North

1.93

$1.59-2.35$

\section{Sex}

Male

Female

1.00

1.57

$1.44-1.70$

\section{Age Group}

18 to 39 years old

40 to 59 years old

1.00

60 years old or more

1.34

$1.20-1.49$

1.42

$1.24-1.63$

\section{Educational level $^{\mathrm{d}}$}

Complete Elementary school

Complete High school

1.00

1.00

$0.88-1.12$

University degree or more

1.99

$1.72-2.30$

\section{Per capita income ${ }^{e}$}

Up to $1 / 2 \mathrm{MW}$

Over $1 / 2 \mathrm{MW}$ and up to $1 \mathrm{MW}$

Over $1 \mathrm{MW}$ and up to $2 \mathrm{MW}$

Over $2 \mathrm{MW}$
1.00

0.89

1.03

1.78
$0.75-1.06$

$0.86-1.24$

$1.44-2.19$

\section{Race}




\section{Self-assessment in health}

Good/very good

1.00

1.59

$1.43-1.77$

Fair/bad/very bad

1.59

$1.43-1.77$

\section{Private Health Insurance}

No

Yes

\section{Access to health services}

No

Yes

a Prevalence considering the complex sample design

b $99 \%$ confidence interval considering the complex sample design

${ }^{\mathrm{c}}$ Adjusted Odds Ratio considering the complex sample design

d The variable education categories "illiterate and incomplete elementary school" were aggregated"

e Per capita income: Up to $\$ 126.46$ (equivalent to Up to $1 / 2$ Minimum Wage -MW), over $\$ 136.46$ and up to $\$ 252.91$ (over $1 / 2 \mathrm{MW}$ and up to $1 \mathrm{MW}$ ) - Over $\$ 252.91$ and up to $\$ 502.8$ (More than $1 \mathrm{MW}$ and up to $2 \mathrm{MW}$ ) - Over $\$ 502.83$ (More than $2 \mathrm{MW}$ ).

f Sample of individuals aged 18 years and older who answered the 2019 National Health Survey individual questionnaire

According to sociodemographic and health characteristics, Table 3 shows the prevalence of the types of CAM most frequently used by the Brazilian population (acupuncture, homeopathy, medicinal plants, meditation, and yoga). The prevalence distribution followed a similar pattern according to the region of residence, being higher in the South and Southeast regions, except medicinal plants, which showed a higher prevalence of use in the North and Northeast regions. We found higher prevalence of CAM use among females, higher income levels, education, and access to health care for all CAMs. Higher prevalence of acupuncture, homeopathy and medicinal plants were also found among individuals aged 60 years and over, and meditation and yoga among younger people (up to 59 years of age). 
Table 3. Prevalence of Complementary and Alternative Medicine (CAM) use, according to sociodemographic characteristics, (Brazil, PNS, 2019) e. 


\begin{tabular}{|c|c|c|c|c|c|}
\hline & Acupuncture & Homeopathy & $\begin{array}{l}\text { Medicinal } \\
\text { Plants }\end{array}$ & Meditation & Yoga \\
\hline Variables & $\begin{array}{l}\text { Prevalence }^{a} \\
\text { (IC99\%) }^{b}\end{array}$ & $\begin{array}{l}\text { Prevalence } \\
\text { (IC99\%) }\end{array}$ & $\begin{array}{l}\text { Prevalence } \\
\text { (IC99\%) }\end{array}$ & $\begin{array}{l}\text { Prevalence } \\
\text { (IC99\%) }\end{array}$ & $\begin{array}{l}\text { Prevalence } \\
\text { (IC99\%) }\end{array}$ \\
\hline \multicolumn{6}{|l|}{ Region } \\
\hline \multirow[t]{2}{*}{ Southeast } & 2.1 & 1.2 & 1.9 & 0.8 & 0.56 \\
\hline & $(1.7-2.4)$ & $(0.9-1.5)$ & $(1.5-2.3)$ & $(0.6-1.1)$ & $(0.4-0.73)$ \\
\hline \multirow[t]{2}{*}{ South } & 1.6 & 1.2 & 3.2 & 0.9 & 0.65 \\
\hline & $(1.3-1.9)$ & $(0.9-1.5)$ & $(2.6-3.8)$ & $(0.7-1.2)$ & $(0.5-0.8)$ \\
\hline \multirow[t]{2}{*}{ Midwest } & 1.1 & 0.9 & 2.6 & 0.7 & 0.36 \\
\hline & $(0.8-1.3)$ & $(0.6-1.1)$ & $(1.9-3.2)$ & $(0.5-0.9)$ & $(0.2-0.5)$ \\
\hline \multirow[t]{2}{*}{ Northeast } & 0.7 & 0.2 & 3.9 & 0.3 & 0.19 \\
\hline & $(0.6-0.9)$ & $(0.2-0.3)$ & $(3.2-4.6)$ & $(0.2-0.4)$ & $(0.1-0.3)$ \\
\hline \multirow[t]{2}{*}{ North } & 0.4 & 0.5 & 5.9 & 0.2 & 0.12 \\
\hline & $(0.3-0.6)$ & $(0.3-0.6)$ & $(5.0-6.9)$ & $(0.1-0.3)$ & $(0.1-0.2)$ \\
\hline \multicolumn{6}{|l|}{ Sex } \\
\hline \multirow[t]{2}{*}{ Male } & 0.9 & 0.5 & 2.4 & 0.4 & 0.2 \\
\hline & $(0.7-1.0)$ & $(0.4-0.6)$ & $(2.1-2.7)$ & $(0.3-0.5)$ & $(0.1-0.2)$ \\
\hline \multirow[t]{2}{*}{ Female } & 1.9 & 1.2 & 3.5 & 0.9 & 0.7 \\
\hline & $(1.7-2.2)$ & $(1.0-1.4)$ & $(3.1-3.8)$ & $(0.7-1.0)$ & $(0.5-0.8)$ \\
\hline \multicolumn{6}{|l|}{ Age Group } \\
\hline \multirow[t]{2}{*}{18 to 39 years old } & 1.0 & 0.7 & 2.3 & 0.7 & 0.5 \\
\hline & $(0.8-1.2)$ & $(0.5-0.9)$ & $(1.9-2.5)$ & $(0.6-0.9)$ & $(0.4-0.6)$ \\
\hline \multirow[t]{2}{*}{40 to 59 years old } & 1.7 & 1.0 & 3.2 & 0.7 & 0.4 \\
\hline & $(1.4-2.0)$ & $(0.8-1.2)$ & $(2.8-3.6)$ & $(0.5-0.8)$ & $(0.3-0.5)$ \\
\hline \multirow{2}{*}{$\begin{array}{l}60 \text { years old or } \\
\text { more }\end{array}$} & 1.9 & 1.0 & 4.0 & 0.5 & 0.3 \\
\hline & $(1.5-2.2)$ & $(0.8-1.2)$ & $(3.5-4.4)$ & $(0.3-0.7)$ & $(0.2-0.8)$ \\
\hline \multicolumn{6}{|l|}{ Educational level $^{\mathrm{C}}$} \\
\hline \multirow{2}{*}{$\begin{array}{l}\text { Complete } \\
\text { Elementary school }\end{array}$} & 0.6 & 0.3 & 3.3 & 0.07 & 0.04 \\
\hline & $(0.5-0.8)$ & $(0.2-0.4)$ & $(2.9-3.7)$ & $(0.0-0.1)$ & $(0.0-0.1)$ \\
\hline
\end{tabular}




\begin{tabular}{|c|c|c|c|c|c|}
\hline \multirow{2}{*}{$\begin{array}{l}\text { Complete High } \\
\text { school }\end{array}$} & 1.0 & 0.5 & 2.1 & 0.28 & 0.17 \\
\hline & $(0.8-1.2)$ & $(0.4-0.7)$ & $(1.8-2.4)$ & $(0.2-0.4)$ & $(0.1-0.2)$ \\
\hline \multirow{2}{*}{$\begin{array}{l}\text { University degree } \\
\text { or more }\end{array}$} & 3.8 & 2.5 & 3.8 & 2.46 & 1.64 \\
\hline & $(3.3-4.3)$ & $(2.1-3.0)$ & $(3.2-4.4)$ & $(2.0-2.9)$ & $(1.3-2.0)$ \\
\hline \multicolumn{6}{|l|}{$\begin{array}{l}\text { Per capita income } \\
d\end{array}$} \\
\hline \multirow[t]{2}{*}{ Up to $1 / 2 \mathrm{MW}$} & 0.2 & 0.2 & 3.3 & 0.10 & 0.034 \\
\hline & $(0.1-0.3)$ & $(0.1-0.3)$ & $(2.7-3.9)$ & $(0.0-0.2)$ & $(0.0-0.1)$ \\
\hline \multirow{2}{*}{$\begin{array}{l}\text { Over } 1 / 2 \mathrm{MW} \text { and } \\
\text { up to } 1 \mathrm{MW}\end{array}$} & 0.5 & 0.3 & 2.6 & 0.19 & 0.081 \\
\hline & $(0.4-0.6)$ & $(0.2-0.5)$ & $(2.2-3.0)$ & $(0.1-0.3)$ & $(0.0-0.1)$ \\
\hline \multirow{2}{*}{$\begin{array}{l}\text { Over } 1 \mathrm{MW} \text { and up } \\
\text { to } 2 \mathrm{MW}\end{array}$} & 1.2 & 0.6 & 2.6 & 0.41 & 0.324 \\
\hline & $(1.0-1.5)$ & $(0.4-0.8)$ & $(2.2-3.0)$ & $(0.2-0.7)$ & $(0.1-0.5)$ \\
\hline \multirow[t]{2}{*}{ Over $2 \mathrm{MW}$} & 4.2 & 2.6 & 3.6 & 2.18 & 1.442 \\
\hline & $(3.6-4.8)$ & $(2.0-3.1)$ & $(3.0-4.1)$ & $(1.8-2.5)$ & $(1.2-1.7)$ \\
\hline \multicolumn{6}{|l|}{ Race } \\
\hline \multirow[t]{2}{*}{ Non-white } & 0.9 & 0.4 & 3.1 & 0.4 & 0.2 \\
\hline & $(0.7-1.0)$ & $(0.3-0.5)$ & $(2.8-3.5)$ & $(0.3-0.4)$ & $(0.1-0.3)$ \\
\hline \multirow[t]{2}{*}{ White } & 2.1 & 1.4 & 2.8 & 1.0 & 0.7 \\
\hline & $(1.8-2.4)$ & $(1.1-1.7)$ & $(2.4-3.2)$ & $(0.8-1.3)$ & $(0.6-0.9)$ \\
\hline \multicolumn{6}{|l|}{$\begin{array}{l}\text { Self-assessment in } \\
\text { health }\end{array}$} \\
\hline \multirow[t]{2}{*}{ Good/very good } & 1.4 & 1.0 & 2.4 & 0.8 & 0.54 \\
\hline & $(1.2-1.6)$ & $(0.8-1.1)$ & $(2.1-2.6)$ & $(0.6-1.0)$ & $(0.4-0.6)$ \\
\hline \multirow[t]{2}{*}{ Fair/bad/very bad } & 1.5 & 0.7 & 4.2 & 0.3 & 0.20 \\
\hline & $(1.2-1.8)$ & $(0.5-0.9)$ & $(3.8-4.7)$ & $(0.2-0.4)$ & $(0.1-0.3)$ \\
\hline \multicolumn{6}{|l|}{$\begin{array}{l}\text { Private Health } \\
\text { Insurance }\end{array}$} \\
\hline \multirow[t]{2}{*}{ No } & 0.6 & 0.4 & 2.9 & 0.3 & 0.19 \\
\hline & $(0.5-0.7)$ & $(0.3-0.5)$ & $(2.6-3.2)$ & $(0.2-0.3)$ & $(0.1-0.2)$ \\
\hline \multirow[t]{2}{*}{ Yes } & 3.7 & 2.1 & 3.2 & 1.7 & 1.15 \\
\hline & $(3.2-4.1)$ & $(1.7-2.5)$ & $(2.7-3.8)$ & $(1.4-2.0)$ & $(0.9-1.4)$ \\
\hline
\end{tabular}




\section{Access to health services}

\begin{tabular}{|c|c|c|c|c|c|}
\hline \multirow[t]{2}{*}{ No } & 0.3 & 0.3 & 2.3 & 0.3 & 0.13 \\
\hline & $(0.2-0.4)$ & $(0.2-0.4)$ & $(1.9-2.7)$ & $(0.2-0.4)$ & $(0.1-0.2)$ \\
\hline \multirow[t]{2}{*}{ Yes } & 1.8 & 1.1 & 3.2 & 0.8 & 0.52 \\
\hline & $(1.6-2.0)$ & $(0.9-1.2)$ & $(2.9-3.5)$ & $(0.6-0.9)$ & $(0.4-0.6)$ \\
\hline \multirow[t]{2}{*}{ Brazil } & 1.4 & 0.9 & 3.0 & 0.7 & 0.4 \\
\hline & $(1.3-1.6)$ & $(0.7-1.0)$ & $(2.7-3.3)$ & $(0.6-0.8)$ & $(0.4-0.5)$ \\
\hline \multicolumn{6}{|c|}{ a Prevalence considering the complex sample design } \\
\hline \multicolumn{6}{|c|}{ b $99 \%$ confidence interval considering the complex sample design } \\
\hline \multicolumn{6}{|c|}{$\begin{array}{l}\text { "The variable education categories "illiterate and incomplete elementary } \\
\text { school" were aggregated" }\end{array}$} \\
\hline \multicolumn{6}{|c|}{$\begin{array}{l}\text { d Per capita income: Up to } \$ 126.46 \text { (equivalent to Up to } 1 / 2 \text { Minimum Wage }-\mathrm{MW} \text { ), } \\
\text { over } \$ 136.46 \text { and up to } \$ 252.91 \text { (over } 1 / 2 \mathrm{MW} \text { and up to } 1 \mathrm{MW} \text { ) - Over } \$ 252.91 \\
\text { and up to } \$ 502.8 \text { (More than } 1 \mathrm{MW} \text { and up to } 2 \mathrm{MW} \text { ) - Over } \$ 502.83 \text { (More than } \\
2 \mathrm{MW} \text { ). }\end{array}$} \\
\hline \multicolumn{6}{|c|}{ e Sample of individuals aged 18 years and older who answered the 2019} \\
\hline
\end{tabular}

The prevalence of use of integrative community therapy was $0.09 \%(0.06-0.15)$, tai-chi (lian gong, qi gong) was $0.06 \%(0.03-0.13)$, and auriculotherapy was $0.35 \%(0.27-0.46)$, non-tabulated data.

White color/race individuals also showed a higher prevalence of CAM use, except for medicinal plants (Table 4). Acupuncture and medicinal plants were frequent among individuals with a report of poor health, while homeopathy, meditation, and yoga were more frequent among those with a report of better health. In the multivariate analyses, the use of CAM was associated with female gender, higher education, older age (except for meditation and yoga), and higher income (except for medicinal plants).

Associations between regions showed different patterns. Using the Southeast region as a reference, residents of the South region had greater chances of using medicinal plants; and those from the Midwest region had lower chances of using acupuncture and a greater chance of using medicinal plants. Residents of the Northeast region had a lower chance of using acupuncture and homeopathy and a greater chance of using medicinal plants. Residents of the North region had a lower chance of using acupuncture, meditation and yoga, and a greater chance of using medicinal plants. There were even greater chances of using homeopathy among white individuals with health insurance and access; greater chances of acupuncture use among individuals with worse self-reported health, who had health insurance and access to health care; greater chances of using medicinal plants among individuals with worse health status; greater chances of using meditation with private health insurance, and greater chances of using yoga among individuals with access to health care (Table 4). 
Table 4. Factors associated with the use of Complementary and Alternative Medicine, (Brazil, PNS-2019). 
Acupuncture Homeopathy Medicinal Meditation Yoga Plants

Prevalence of CAM use ${ }^{a}$ $(99 \% \mathrm{Cl})^{\mathrm{b}}$ by sociodemographic variables
$\mathrm{AOR}^{\mathrm{c}}$

$(99 \% \mathrm{Cl})$
AOR

$(99 \% \mathrm{Cl})$
AOR

$(99 \% \mathrm{Cl})$
AOR

$(99 \% \mathrm{Cl})$
AOR

$(99 \% \mathrm{Cl})$

\section{Region}

\begin{tabular}{llllll} 
Southeast & 1.0 & 1.0 & 1.0 & 1.0 & 1.0 \\
South & 0.80 & 0.96 & $\mathbf{1 . 7 7}$ & 1.17 & 1.18 \\
& $(0.61-1.04)$ & $(0.68-1.37)$ & $\mathbf{( 1 . 3 3 -}$ & $(0.82-$ & $(0.79-$ \\
Midwest & & & $\mathbf{2 . 3 7}$ & $1.66)$ & $1.75)$ \\
& $\mathbf{0 . 6 1}$ & 0.84 & $\mathbf{1 . 4 2}$ & 0.92 & 0.73 \\
& $(\mathbf{0 . 4 5 - 0 . 8 3 )}$ & $(0.57-1.23)$ & $\mathbf{( 1 . 0 2 -}$ & $(0.61-$ & $(0.46-$ \\
Northeast & $\mathbf{0 . 6 6}$ & $\mathbf{0 . 3 4}$ & $\mathbf{2 . 2 4}$ & 0.73 & 0.72 \\
& $(\mathbf{0 . 4 9 - 0 . 8 9 )}$ & $\mathbf{( 0 . 2 3 - 0 . 5 0 )}$ & $\mathbf{( 1 . 6 9 -}$ & $(0.50-$ & $(0.43-$ \\
& & & $\mathbf{2 . 9 6 )}$ & $1.09)$ & $1.21)$ \\
\hline North & $\mathbf{0 . 4 2}$ & 0.73 & $\mathbf{3 . 6 5}$ & $\mathbf{0 . 4 4}$ & $\mathbf{0 . 4 7}$ \\
& $\mathbf{( 0 . 3 0 - 0 . 5 8 )}$ & $(0.48-1.10)$ & $\mathbf{( 2 . 8 1 -}$ & $\mathbf{( 0 . 2 6 -}$ & $\mathbf{0 . 2 5 -}$ \\
& & & $\mathbf{4 . 7 5 )}$ & $\mathbf{0 . 7 2 )}$ & $\mathbf{0 . 8 6})$
\end{tabular}

Sex

$\begin{array}{llllll}\text { Male } & 1.0 & 1.0 & 1.0 & 1.0 & 1.0 \\ \text { Female } & \mathbf{1 . 9 7} & \mathbf{2 . 0 5} & \mathbf{1 . 3 6} & \mathbf{1 . 9 5} & \mathbf{3 . 6 3} \\ & (\mathbf{1 . 6 2 - 2 . 3 9 )} & \mathbf{( 1 . 6 9 - 2 . 4 9 )} & \mathbf{( 1 . 2 4 -} & \mathbf{( 1 . 5 2 -} & \mathbf{( 2 . 4 9 -} \\ & & & \mathbf{1 . 4 9 )} & \mathbf{2 . 5 1 )} & \mathbf{5 . 2 8 )}\end{array}$

\section{Age Group}

18 to 39 years old

1.0

40 to 59 years old

60 years old or more

1.53

(1.20-1.96)

1.56

(1.18-2.06)
1.0

1.34

(1.04-1.73)

1.35

(0.96-1.88)
1.0

1.35

(1.19-

1.54)

1.59

(1.33-

1.90)
1.0

0.95

(0.67-

1.36)

0.91

(0.561.47)
1.0

0.72

(0.48-

1.07)

0.79

(0.441.43) 
Complete Elementary school

$\begin{array}{lllll}1.0 & 1.0 & 1.0 & 1.0 & 1.0\end{array}$

Complete High school

1.51

1.58

0.85

2.99

2.60

$(1.06-2.14)$

(1.11-2.26)

(0.74-

$0.98)$

$(1.63-$

$5.49)$

$(1.24-$

University degree or more

2.90
$(2.01-4.17)$

4.00

1.49

13.48

11.13

(2.70-5.92)

(1.26-

(6.41-

28.33)

(4.51-

27.44)

\section{Per capita income ${ }^{\mathrm{e}}$}

\begin{tabular}{|c|c|c|c|c|c|}
\hline Up to $1 / 2 \mathrm{MW}$ & 1.0 & 1.0 & 1.0 & 1.0 & 1.0 \\
\hline \multirow[t]{2}{*}{ Over $1 / 2 \mathrm{MW}$ and up to $1 \mathrm{MW}$} & 1.55 & 1.11 & 0.85 & 1.24 & 1.58 \\
\hline & $(0.95-2.53)$ & $(0.60-2.08)$ & $\begin{array}{l}(0.69- \\
1.03)\end{array}$ & $\begin{array}{l}(0.60- \\
2.60)\end{array}$ & $\begin{array}{l}(0.62- \\
3.99)\end{array}$ \\
\hline \multirow[t]{2}{*}{ Over $1 \mathrm{MW}$ and up to $2 \mathrm{MW}$} & 2.69 & 1.27 & 0.94 & 1.49 & 3.59 \\
\hline & $(1.66-4.17)$ & $(0.68-2.36)$ & $\begin{array}{l}(0.75- \\
1.19)\end{array}$ & $\begin{array}{l}(0.66- \\
3.35)\end{array}$ & $\begin{array}{l}(1.38- \\
9.29)\end{array}$ \\
\hline \multirow[t]{2}{*}{ Over $2 \mathrm{MW}$} & 5.16 & 2.78 & 1.17 & 3.63 & 7.50 \\
\hline & $(2.93-9.08)$ & $(1.47-5.25)$ & $\begin{array}{l}(0.92- \\
1.49)\end{array}$ & $\begin{array}{l}(1.66- \\
7.93)\end{array}$ & $\begin{array}{l}(2.97- \\
18.93)\end{array}$ \\
\hline
\end{tabular}

\section{Race}

Non-white

1.0

1.0

1.0

1.0

1.0

White

1.15

1.51

0.96

1.20

1.36

(0.90-1.45)

(1.16-1.96)

(0.84-

(0.88-

1.66)

(0.90-

2.06)

\section{Self-assessment in health}

\begin{tabular}{llllll} 
Good/very good & 1.0 & 1.0 & 1.0 & 1.0 & 1.0 \\
\hline Fair/bad/very bad & 1.90 & 1.37 & 1.63 & 0.90 & 1.02 \\
& $(\mathbf{1 . 5 1 - 2 . 3 9 )}$ & $(1.02-1.83)$ & $\begin{array}{l}(\mathbf{1 . 4 3 -} \\
\mathbf{1 . 8 5})\end{array}$ & $\begin{array}{l}(0.66- \\
1.23)\end{array}$ & $\begin{array}{c}(0.62- \\
3.99)\end{array}$ \\
\hline Private Health Insurance & & & & & \\
\hline No & 1.0 & 1.0 & 1.0 & 1.0 & 1.0 \\
Yes & $\mathbf{2 . 2 4}$ & $\mathbf{1 . 5 0}$ & 1.13 & $\mathbf{1 . 4 3}$ & 1.47 \\
& $(\mathbf{1 . 6 7 - 3 . 0 1 )}$ & $\mathbf{( 1 . 0 6 - 2 . 1 3 )}$ & $(0.93-$ & $\mathbf{( 0 . 9 9 -}$ & $(0.85-$ \\
& & & $1.35)$ & $\mathbf{2 . 0 5 )}$ & $2.55)$
\end{tabular}




\begin{tabular}{llllll}
\hline No & 1.0 & 1.0 & 1.0 & 1.0 & 1.0 \\
\hline Yes & $\mathbf{2 . 5 9}$ & $\mathbf{1 . 9 5}$ & 1.14 & 1.40 & $\mathbf{1 . 8 2}$ \\
& $\mathbf{( 1 . 7 5 - 3 . 8 5 )}$ & $\mathbf{( 1 . 3 0 - 2 . 9 2 )}$ & $(0.97-$ & $(0.91-$ & $\mathbf{( 1 . 0 5 -}$ \\
& & & $1.34)$ & $\mathbf{2 . 1 6 )}$ & $\mathbf{3 . 1 4 )}$ \\
\hline
\end{tabular}

${ }^{a}$ Prevalence considering the complex sample design

b $99 \%$ confidence interval considering the complex sample design

${ }^{\mathrm{c}}$ Adjusted Odds Ratio considering the complex sample design

d The variable education categories "illiterate and incomplete elementary school" were aggregated"

e Per capita income: Up to $\$ 126.46$ (equivalent to Up to $1 / 2$ Minimum Wage -MW), over $\$ 136.46$ and up to $\$ 252.91$ (over $1 / 2 \mathrm{MW}$ and up to $1 \mathrm{MW}$ ) - Over $\$ 252.91$ and up to $\$ 502.8$ (More than $1 \mathrm{MW}$ and up to $2 \mathrm{MW}$ ) - Over $\$ 502.83$ (More than $2 \mathrm{MW}$ ).

${ }^{f}$ Sample of individuals aged 18 years and older who answered the 2019 National Health Survey individual questionnaire

Table 5 shows the prevalence of acupuncture, homeopathy, medicinal plants, and the use of at least one CAM for the years 2013 and 2019. The primary trend is an increase in prevalence among the sociodemographic variables evaluated in the period. However, there is stability in the prevalence of CAM in the South and homeopathy in the Northeast region. It is also observed that there is stability in the use of CAMs among individuals with lower educational levels and the use of homeopathy among those of non-white color/race. Homeopathy and all CAM use decreased in the South region. There was also a decrease in the prevalence of medicinal plants use in the South and North region. There was also a slight decrease in the use of medicinal plants among non-white individuals (Table 5).

Table 5. Evolution of the prevalence of CAM use (Acupuncture, Homeopathy, Medicinal Plants, and all CAM) according to sociodemographic characteristics for the years 2013 and 2019 (PNS,2013 and PNS,2019) ${ }^{d}$. 
Acupuncture

Homeopathy

Medicinal

All CAM

Plants

Prevalence $^{\mathrm{a}}(95 \% \mathrm{Cl})^{\mathrm{b}} \quad \begin{aligned} & \text { Prevalence } \\ & (95 \% \mathrm{Cl})\end{aligned}$

Prevalence

(95\% Cl)

Prevalence

(95\% Cl)

\begin{tabular}{lllllllll} 
Year & $\mathbf{2 0 1 3}$ & $\mathbf{2 0 1 9}$ & $\mathbf{2 0 1 3}$ & $\mathbf{2 0 1 9}$ & $\mathbf{2 0 1 3}$ & $\mathbf{2 0 1 9}$ & $\mathbf{2 0 1 3}$ & $\mathbf{2 0 1 9}$ \\
Region & & & & & & & & \\
Southeast & 1.5 & 2.0 & 0.6 & 1.2 & 1.3 & 1.9 & 3.8 & 5.0 \\
& $(1.3-$ & $(1.8-2.3)$ & $(0.5-$ & $(1.0-$ & $(0.9-$ & $(1.6-$ & $(3.2-4.5)$ & $(4.5-$ \\
& $1.9)$ & & $0.9)$ & $1.5)$ & $1.9)$ & $2.2)$ & & $5.5)$ \\
\hline South & 1.0 & 1.6 & 1.0 & 1.2 & 3.6 & 3.2 & 6.1 & 6.1 \\
& $(0.7-$ & $(1.3-1.8)$ & $(0.7-$ & $(1.0-$ & $(2.8-$ & $(2.7-$ & $(5.1-7.3)$ & $(5.5-$ \\
& $1.4)$ & & $1.6)$ & $1.4)$ & $4.7)$ & $3.6)$ & & $6.7)$ \\
\hline Midwest & 0.7 & 1.1 & 0.7 & 0.9 & 2.5 & 2.6 & 4.1 & 4.2 \\
& $(0.5-$ & $(0.9-1.3)$ & $(0.5-$ & $(0.7-$ & $(2.0-$ & $(2.1-$ & $(3.5-4.9)$ & $(3.7-$ \\
& $0.9)$ & & $1.0)$ & $1.1)$ & $3.2)$ & $3.0)$ & & $4.8)$ \\
\hline Northeast & 0.3 & 0.7 & 0.2 & 0.2 & 3.5 & 3.9 & 4.1 & 4.9 \\
& $(0.2-$ & $(0.6-0.9)$ & $(0.1-$ & $(0.2-$ & $(2.9$ & $(3.3$ & $(3.5-4.8)$ & $(4.3-$ \\
& $0.4)$ & & $0.3)$ & $0.3)$ & $-4.2)$ & $-4.5)$ & & $5.5)$ \\
\hline \multirow{2}{*}{ North } & 0.1 & 0.4 & 0.6 & 0.5 & 6.2 & 5.9 & 7.0 & 6.6 \\
& $(0.1-$ & $(0.4-0.5)$ & $(0.4-$ & $(0.4-$ & $(4.9-$ & $(5.2-$ & $(5.8-8.5)$ & $(5.9-$ \\
& $0.3)$ & & $0.8)$ & $0.6)$ & $7.7)$ & $6.6)$ & & $7.4)$
\end{tabular}

Sex

$\begin{array}{lllllllll}\text { Male } & 0.6 & 0.9 & 0.5 & 0.5 & 2.4 & 2.4 & 3.7 & 3.8 \\ & (0.5- & (0.8-1.0) & (0.3- & (0.4- & (2.1- & (2.2- & (3.3-4.2) & (3.5- \\ & 0.9) & & 0.6) & 0.6) & 2.8) & 2.6) & & 4.1) \\ \text { Female } & 1.3 & 1.9 & 0.7 & 1.2 & 2.9 & 3.5 & 5.2 & 6.4 \\ & (1.1- & (1.7-2.1) & (0.6- & (1.0- & (2.6- & (3.2- & (4.7-5.7) & (6.1- \\ & 1.5) & & 0.8) & 1.3) & 3.4) & 3.7) & & 6.8)\end{array}$

\section{Age Group}

old

$0.5 \quad 1.0$

$\begin{array}{ll}0.5 & 0.7\end{array}$

2.1

2.2

3.4

4.1

(0.4- $\quad(0.8-1.1)$

(0.4- (0.6-

(1.8-

(2.0-

2.5) 2.5)

(3.0-3.9)

(3.8-

0.7) 0.8$)$

3.1

3.2

5.5

5.8 
old
$(1.0-\quad(1.5-1.9)$
(0.5- (0.8-
(2.6-
(2.9-
$(4.9-6.2)$
$(5.4-$
1.7)
0.9) 1.2)
3.6) 3.5)
$6.2)$

60 years old or $\quad 1.4 \quad 1.9$

$\begin{array}{lllll}0.5 & 1.0 & 3.4 & 4.0 & 5.4\end{array}$

6.5

more
$(1.1-$
$1.7)$
$(1.6-2.2)$
(0.3-
(0.8-
$\begin{array}{ll}(2.9- & (3.6- \\ 4.0) & 4.3)\end{array}$
$(4.7-6.1)$
(6.0-
7.0)

\section{Educational}

level $^{\mathrm{c}}$

\begin{tabular}{|c|c|c|c|c|c|c|c|c|}
\hline \multirow{2}{*}{$\begin{array}{l}\text { Complete } \\
\text { Elementary } \\
\text { school }\end{array}$} & 0.3 & 0.6 & 0.3 & 0.3 & 3.5 & 3.3 & 4.2 & 4.2 \\
\hline & $\begin{array}{l}(0.2- \\
0.5)\end{array}$ & $(0.5-0.8)$ & $\begin{array}{l}(0.2- \\
0.4)\end{array}$ & $\begin{array}{l}(0.3- \\
0.4)\end{array}$ & $\begin{array}{l}(2.9- \\
4.1)\end{array}$ & $\begin{array}{l}(3.0- \\
3.6)\end{array}$ & $(3.6-4.9)$ & $\begin{array}{l}(3.9- \\
4.5)\end{array}$ \\
\hline \multirow{2}{*}{$\begin{array}{l}\text { Complete High } \\
\text { school }\end{array}$} & 0.8 & 1.0 & 0.4 & 0.5 & 1.9 & 2.1 & 3.3 & 3.7 \\
\hline & $\begin{array}{l}(0.6- \\
1.0)\end{array}$ & $(0.8-1.1)$ & $\begin{array}{l}(0.3- \\
0.6)\end{array}$ & $\begin{array}{l}(0.4- \\
0.6)\end{array}$ & $\begin{array}{l}(1.6- \\
2.2)\end{array}$ & $\begin{array}{l}(1.9- \\
2.3)\end{array}$ & $(3.0-3.7)$ & $\begin{array}{l}(3.4- \\
4.0)\end{array}$ \\
\hline \multirow{2}{*}{$\begin{array}{l}\text { University } \\
\text { degree or more }\end{array}$} & 3.2 & 3.8 & 1.8 & 2.5 & 2.4 & 3.8 & 8.1 & 9.9 \\
\hline & $\begin{array}{l}(2.6- \\
3.9)\end{array}$ & $(3.4-4.2)$ & $\begin{array}{l}(1.4- \\
2.3)\end{array}$ & $\begin{array}{l}(2.2- \\
2.9)\end{array}$ & $\begin{array}{l}(1.9- \\
2.9)\end{array}$ & $\begin{array}{l}(3.3- \\
4.2)\end{array}$ & $(7.2-9.1)$ & $\begin{array}{l}(9.2- \\
10.6)\end{array}$ \\
\hline
\end{tabular}

\section{Race}

\begin{tabular}{|c|c|c|c|c|c|c|c|c|}
\hline \multirow[t]{2}{*}{ Non-white } & 0.5 & 0.9 & 0.4 & 0.4 & 3.2 & 3.1 & 4.3 & 4.4 \\
\hline & $\begin{array}{l}(0.4- \\
0.7)\end{array}$ & $(0.8-1.0)$ & $\begin{array}{l}(0.3- \\
0.5)\end{array}$ & $\begin{array}{l}(0.4- \\
0.5)\end{array}$ & $\begin{array}{l}(2.8- \\
3.8)\end{array}$ & $\begin{array}{l}(2.8- \\
3.4)\end{array}$ & $(3.7-4.9)$ & $\begin{array}{l}(4.1- \\
4.7)\end{array}$ \\
\hline \multirow[t]{2}{*}{ White } & 1.5 & 2.1 & 0.8 & 1.4 & 2.1 & 2.8 & 4.7 & 6.2 \\
\hline & $\begin{array}{l}(1.2- \\
1.7)\end{array}$ & $(1.9-2.4)$ & $\begin{array}{l}(0.7- \\
1.0)\end{array}$ & $\begin{array}{l}(1.2- \\
1.6)\end{array}$ & $\begin{array}{l}(1.8- \\
2.4)\end{array}$ & $\begin{array}{l}(2.5- \\
3.1)\end{array}$ & $(4.3-5.2)$ & $\begin{array}{l}(5.8- \\
6.7)\end{array}$ \\
\hline \multirow[t]{3}{*}{ Brazil (total) } & 1,0 & 1,5 & 0,6 & 1,0 & 2,7 & & 4,5 & 5,2 \\
\hline & $\begin{array}{l}(0,8- \\
1,1)\end{array}$ & & $\begin{array}{l}(0,5- \\
0,7)\end{array}$ & & $\begin{array}{l}(2,4- \\
3,0)\end{array}$ & $\begin{array}{l}(3,0- \\
3,4)\end{array}$ & $(4,1-4,9)$ & $\begin{array}{l}(5,1- \\
5,8)\end{array}$ \\
\hline & & $(1,4-1,7)$ & & $\begin{array}{l}(0,9-9 \\
1,1)\end{array}$ & & & & \\
\hline
\end{tabular}

a Prevalence considering the complex sample design

b $95 \%$ confidence interval considering the complex sample design 
c The variable education categories "illiterate and incomplete elementary school" were aggregated"

d Sample of individuals aged 18 years and older who answered the 2013 and 2019 National Health Survey individual questionnaires

\section{Discussion}

About 8,500,000 Brazilian adults over 18 years old reported using one or more CAM in 2019, equivalent to $5.2 \%$ of the country's population, an increase of $0.7 \%$ in the prevalence of CAM use compared to the PNS2013 research. Medicinal plants were the most frequently used CAMs in Brazil, with substantial regional differences: while in the Southeast, a more industrialized and urbanized Brazilian region, the use of acupuncture was more frequent, in the Northeast and North regions, that contains most of the Amazon Forest, the use of medicinal plants was more frequently reported by the population. This heterogeneity was maintained when comparing these findings with those of the PNS-2013.

Brazil has five regions, with significant sociodemographic and health differences between them. The South and Southeast regions are the most densely populated and economically developed, with better health indicators, better access to health services ${ }^{15}$, and longer life expectancy ${ }^{16}$ than the North, Northeast, and Midwest regions. The North region concentrates more than $80 \%$ of the indigenous population ${ }^{17}$, likely influencing the higher prevalence of medicinal plants. Paradoxically, the North region has the lowest offer of CAM in primary health care ${ }^{18}$. Indigenous practices and their cosmological perspectives are not incorporated into the National Policy on Integrative and Complementary Health Practices of the Unified Health System and other famous practices such as prayers, healers, and midwives ${ }^{19}$. In Brazil, the discussion about the insertion of these traditional practices into policies is still incipient and has not received adequate research support, so we do not know what percentage of the population seeks this type of care ${ }^{19}$. We can assume that if these practices were incorporated into the PNS questionnaire, the prevalence of CAM would be even higher in this region.

The present study also observed that female individuals with complete higher education, per capita family income higher than two minimum wages (more than \$502.83), and aged 40 years or more were more likely to use one or more CAM. This profile of CAM use is similar to that observed in another studies 20-22. According to the PNS 2019, the demand for health services, in general, was higher among older women with a high level of education ${ }^{23}$. CAMs are also used as a therapeutic alternative and provide, in addition to physical relief, an improvement in quality of life, well-being, and anxiety control ${ }^{24,25}$. Studies show that many women would like to avoid pharmacological or invasive pain management methods, especially during childbirth, encouraging them to use non-biomedical practices such as acupuncture ${ }^{26}$. Some studies have also shown that women use integrative practices at different stages of life, such as during pregnancy, postpartum to climacteric, and menopause 27,28 .

The present study observed that individuals with better access to health care are more likely to use CAM, and in the adjusted analysis, it was possible to observe that these individuals were more likely to use 
acupuncture, homeopathy, and yoga, respectively. These findings suggest possible inequalities in access to health services, as these practices require specialized professionals who might not be available in the Brazilian Unified Health System. In the PNS -2013, it was observed that acupuncture and homeopathy are among the most used CAMs by those who had a private health plan ${ }^{10}$. In PNS- 2019, it was not possible to analyze the use of CAM through a private, out-of-pocket, or health plan.

The federal government adopted The Family Health Strategy to expand Primary Health Care in Brazil, covering $65 \%$ of the country's population ${ }^{29}$. Brazil's Family Health Strategy has been offered most CAM, despite the disparity between the Ministry of Health and independent surveys ${ }^{29,30,18}$. Studies observed that some Family Health Strategy professionals offer CAM in the Unified Health System by their initiative and expenses.

There is still a mismatch between the supply and use of CAM, especially within the Unified Health System. The Ministry of Health of Brazil ${ }^{30}$ reports an increase in the supply of CAM in the Unified Health System across the country. However, the use of CAM through the Unified Health System is still relatively low $^{18,6,10}$. Studies have shown that the offer in Brazil is dependent on health professionals; that is, there is no specific financing, and the offer depends most on the willingness of the health professional to offer CAM services ${ }^{18}$. Thus, practices that require specific inputs (such as acupuncture) and higher specialization, such as Acupuncture and Homeopathy (a specialty restricted to medical activity in Brazil), continue to be offered less, even after 15 years of National Policy on Integrative and Complementary Practices implementation ${ }^{18}$.

Regarding self-rated health, the present survey observed that individuals who reported having regular, poor, or very poor health are more likely to use CAM compared to those who reported having better health status. In the evaluation by type of CAM, it was also possible to observe that those who declared a worse health situation are more likely to use acupuncture or medicinal plants. Similar results associating worse health status and greater use of CAM were observed in other studies $31,21,12$. Having a private health insurance plan also increased the chances of using CAM, and similar findings were found in other surveys $32,33,20$.

Meditation, yoga, tai chi chuan (or lian gong or qi gong), integrative community therapy, and auriculotherapy are CAM that was not included in the PNS- 2013 and were included in 2019 after insertion in the National Policy on Integrative and Complementary Practices. The present study observed a low prevalence of tai chi chuan (or lian gong or qi gong), and integrative community therapy. Meditation and yoga also had a low prevalence use among Brazilian adults. A population survey conducted in the United States found a prevalence of $8.9 \%$ in yoga among adults, but in the United Kingdom, this prevalence was lower at $1.1 \% 34,35$. Regarding meditation, a survey conducted in the United States found a prevalence of $18.6 \%$ in adults $^{36}$.

The yoga and meditation profile practice stood out in the PNS-2019 among female individuals with private health insurance, access to health services, and reported better self-rated health. Individuals with 
complete higher education were more than ten times more likely to use these two CAM types than others. We can observe that these are types of CAM used by individuals with higher incomes. Similar results for yoga were observed in studies conducted in the United States, the United Kingdom, and Germany $37,35,34$.

In Brazil, studies about the prevalence of the practice of yoga and meditation are still scarce; however, these practices are understood as a form of health promotion, disease prevention, and even as a therapeutic action, thus being adopted by the Unified Health System within the scope of primary care in health ${ }^{38}$. Yoga, tai chi, meditation, among others, have been offered in the Brazilian health system as a set called bodily practices, which can confuse the population in general, as there is not always a distinction about each of them. However, this is the fastest-growing supply in the country, with a more than $200 \%$ increase in the number of services and the one with the highest number of services offered per population 2.20/100000 inhabitant ${ }^{6}$. Studies have shown that yoga and meditation have also been presented as spiritual practices, used mainly for people affected by cancer and other chronic conditions to promote health and quality of life ${ }^{39,40}$.

An extensive systematic review reported a variability by which CAMs are defined and classified in studies $^{9}$, making it difficult to compare the use of these practices across populations. The type of sampling and the target population is a factor that can influence research results. Several studies focus on specific populations or subjects, which can generate an information bias. An example is the high prevalence of CAM use in studies with individuals with specific illnesses (especially chronic or terminal illnesses), while we find lower prevalence in population-based studies such as the Brazilian PNS ${ }^{41-43}$.

Information bias, resulting from the way information about CAMs was collected, may have influenced the results of this study as respondents may not remember any use of CAM or even not consider such practice or therapy to be $\mathrm{CAM}^{44,45}$. In the PNS-2019, a "filter" question was asked in which the respondent had the option of answering "yes" or "no" for the use of eight CAMs previously listed, and if the answer was "yes" to the use of these CAMs, the subsequent questions about each of the eight CAMs were carried out separately. This procedure may have underestimated the prevalence of CAM use in Brazil, which could have been avoided if all eight CAMs selected by the research coordination were read in sequence, allowing the respondent to respond positively or negatively to each type of CAM separately. However, as Unified Health System offers 29 types of CAM, we believe that including all of them in the survey would possibly reduce the information bias, which could reflect a higher prevalence of the use of these therapies.

Another limitation of the study was the non-assessment of the out-of-pocket amount for the use of CAM. Unlike the PNS-2013 analyses, it was impossible to know whether the individual used CAM by Unified Health System, health insurance, or private individuals. The PNS -2019 questionnaire did not cover this information. On the other hand, the strength of the research was its population representation, having reached adults from all socioeconomic strata and allowing the generalization of the results.

\section{Conclusion}


The use of CAM is heterogeneous, with essential differences in the patterns of use between Brazilian regions. Cultural issues can explain these differences and aspects related to the supply and access to health services that provide CAM. Wealthier populations with higher education and access to private health plans use CAM more often that depend on supplies and specialized health professionals, such as acupuncture and homeopathy. The poorest part of the population and with less education use medicinal herbs and herbal medicines more frequently, which have easier access and can often be used without the guidance of health professionals.

The National Policy on Integrative and Complementary Practices in Health has a vital role in disseminating, availability, and promoting a greater variety of CAM use, especially within the Unified Health System, but it does not define what actions and resources are necessary to make this possible. The National Policy on Integrative and Complementary Practices in Health also does not provide the necessary tools for evaluating their implementation, making their implementation, under such conditions, a challenge for public managers. Another point is the challenge of developing more studies on the quality and safety of these practices, requiring more evaluative studies and the effectiveness of some of the practices offered.

\section{Abbreviations}

TCIM - Traditional, Complementary and Integrative Medicine

CAM - Complementary and Alternative Medicine - PIC - Práticas Integrativas e Complementares em Saúde

WHO - World Health Organization

UHS - Unified Health System - SUS - Sistema Único de Saúde -

NPICP - National Policy on Integrative and Complementary Practices in Health - PNPIC - Política Nacional de Práticas Integrativas e Complementares em Saúde

PNS - National Health Survey (Pesquisa Nacional de Saúde)

PAU - Primary Analysis Units

IBGE - Brazilian Institute of Geography and Statistics (Instituto Brasileiro de Geografia e Estatística.)

OR - Odd Ratio

AOR - Ajusted Odd Ratio

Cl - Confidence Interval

\section{Declarations}




\section{Ethics approval and consent to participate}

The data used in the study was in accordance with the relevant guidelines and regulations. The 2019 National Health Survey project was submitted to the National Research Ethics Commission (CONEP)/National Health Council (CNS) and approved under Opinion number. 3.529.376, issued on August 23, 2019.

The dataset used in this study was obtained from the Brazilian Ministry of Health open-data reports https://www.pns.icict.fiocruz.br/bases-de-dados/ with anonymized secondary data. The Ministry of Health of Brazil is committed to respecting the ethical precepts and guaranteeing the privacy and reliability of the data.

\section{Consent for Publication}

Since the analysis was made with secondary anonymized data, consent for publication is not applicable.

\section{Availability of Data and Material (ADM)}

The datasets generated and/or analyzed during the current study are available in the PNS 2019 - Base de Dados Fiocruz, repository, https://www.pns.icict.fiocruz.br/bases-de-dados/.

\section{Competing Interest}

The authors have no competing interests as defined by BMC, or other interests that might be perceived to influence the results and/or discussion reported in this paper.

\section{Funding}

No funding.

\section{Author's Contributions}

PMMB, KLSB and IMCS wrote the main manuscript text and prepared tables; CSB, contribute with the analysis and all authors reviewed the manuscript.

\section{Acknowledgement}

Not applicable.

\section{References}

1. Hawk C, Adams J, Hartvigsen J. The Role of CAM in Public Health, Disease Prevention, and Health Promotion. Evid Based Complement Alternat Med. 2015;2015:528487. 
2. Rhee TG, Harris IM. Reasons for and perceived benefits of utilizing complementary and alternative medicine in U.S. adults with migraines/severe headaches. Complementary Therapies in Clinical Practice. 2018; 30:44-49. https://doi.org/10.1016/j.ctcp.2017.12.003

3. Galante J, Dufour G, Vainre M, Wagner AP, Jan Stochl, Benton A, et al. A mindfulness-based intervention to increase resilience to stress in university students (the Mindful Student Study): a pragmatic randomised controlled trial. Lancet Public Health. 2018;3(2):e72-e81. https://doi.org/10.1016/S2468-2667(17)30231-1

4. Rhee TG, Pawloski PA, Parsons HM. Health-related Quality of Life among US Adults with Cancer: Potential Roles of Complementary and Alternative Medicine for Health Promotion and Well-being. Psychooncology. 2019; 28(4): 896-902. https://doi.org/10.1002/pon.5039

5. World Health Organization (WHO). General guidelines for methodologies on research and evaluation of traditional medicine. Geneva: WHO; 2000. Organization. https://apps.who.int/iris/handle/10665/66783

6. Sousa IMC, Bezerra, AFB, Guimarães MB, Benevides IA, Tesser CD. Traditional, Complementary and Integrative Medicine in the Brazilian Public Health Service: Opportunities and Limitations. Public Health and Health Services Research in Traditional, Complementary and Integrative Health Care. 2019: 197-216 https://doi.org/10.1142/9781786346797_0012

7. BRAZIL. Ministry of Health. National Policy for Complementary and Integrative Practices. Ordinance n. 971. Brasília: Brazilian Federal Register, n. 84, section l; 2006. p. 20-4.

8. Brazil. Ministry of Health. Health Care Secretariat. Ordinance n. 702, of March 21, 2018. It alters the consolidation ordinance n. 2/GM/MS, of September 28, 2017, to include new practices into the National Policy for Complementary and Integrative Practices - PNPIC Brazilian Federal Register 2018.

9. Harris PE, Cooper KL, Relton C, Thomas KJ. Prevalence of complementary and alternative medicine (CAM) use by the general population: a systematic review and update. Int J Clin Pract. 2012;66(10):924-39. https://doi.org/10.1111/j.1742-1241.2012.02945.x

10. Boccolini PMM, Boccolini CS. Prevalence of complementary and alternative medicine (CAM) use in Brazil. BMC Complement Med Ther. 2020; 20: 51. https://doi.org/10.1186/s12906-020-2842-8

11. Clarke TC, Barnes PM, Black LI, Stussman BJ, Nahin RL. Use of Yoga, Meditation, and Chiropractors Among U.S. Adults Aged 18 and Over. NCHS Data Brief. 2018;(325):1-8.

12. Kemppainen LM, Kemppainen TT, Reippainen JA, Salmenniemi ST, Vuolanto PH. Use of complementary and alternative medicine in Europe: Health-related and sociodemographic determinants. Scand J Public Health. 2018;46(4):448-

455. https://doi.org/10.1177/1403494817733869

13. Siti ZM, Tahir A, Farah Al, Fazlin SMA, Sondi S, Azman AH, et al. Use of traditional and complementary medicine in Malaysia: a baseline study. Complement Ther Med. 2009; 17(5-6): 2929. https://doi.org/10.1016/j.ctim.2009.04.002 
14. Sistema Integrado de Pesquisas Domiciliares (SIPD). Ministério do Planejamento, Orçamento e Gestão / Instituto Brasileiro de Geografia e Estatística - IBGE. Pesquisa Nacional de Saúde (2019). Available at: https://www.ibge.gov.br/estatisticas/sociais/saude/9160-pesquisa-nacional-desaude. $h t m l ?=\& t=$ microdados. Acessed 28 Oct 2021.

15. Viacava F, Porto SM, Carvalho CC, Bellido JG. Desigualdades regionais e sociais em saúde segundo inquéritos domiciliares (Brasil, 1998-2013). Ciência \& Saúde Coletiva. 2019; 24(7). https://doi.org/10.1590/1413-81232018247.15812017

16. Camargos MCS, Gonzaga MR, Costa JV, Bomfim WC. Estimativas de expectativa de vida livre de incapacidade funcional para Brasil e Grandes Regiões, 1998 e 2013. Ciência \& Saúde Coletiva. 2019;24(3):737-747. https://doi.org/10.1590/1413-81232018243.07612017

17. Instituto Brasileiro de Geografia e Estatística (IBGE). Os indígenas no Censo Demográfico 2010, primeiras considerações com base no quesito cor ou raça. Rio de Janeiro: IBGE;2012.

18. Barbosa FES, Guimarães MBL, Santos CR, Benjamin AF, Dalcanale BC. Oferta de Práticas Integrativas e Complementares em Saúde na Estratégia Saúde da Família no Brasil. Cadernos de Saúde Pública.2020;36(1). https://doi.org/10.1590/0102-311X00208818

19. Guimarães MB, Nunes JA, Velloso M, Bezerra A, Sousa MII. As práticas integrativas e complementares no campo da saúde: para uma descolonização dos saberes e práticas. Saúde e Sociedade.2020;29(1):e190297. https://doi.org/10.1590/S0104-12902020190297

20. Conboy L, Patel S, Kaptchuk TJ, Gottlieb B, Eisenberg D, Acevedo-Garcia D. Sociodemographic determinants of the utilization of specific types of complementary and alternative medicine: an analysis based on a nationally representative survey sample. J Altern Complement Med. 2005;11(6):977-94.

21. Klein SD, Torchetti L, Frei-Erb M, Wolf U. Usage of Complementary Medicine in Switzerland: Results of the Swiss Health Survey 2012 and Development Since 2007. PLoS One. 2015;10(10):e0141985.

22. Reid R, Steel A, Wardle J, Trubody A, Adams J. Complementary medicine use by the Australian population: a critical mixed studies systematic review of utilisation, perceptions and factors associated with use. BMC Complement Altern Med. 2016; 16: 176. https://doi.org/10.1186/s12906016-1143-8

23. Malta DC, Bernal RTI, Gomes CS, Cardoso LCM, Lima MG, Barros MBA. Desigualdades na utilização de serviços de saúde por adultos e idosos com e sem doenças crônicas no Brasil, Pesquisa Nacional de Saúde 2019 [preprint] Rev Bras Epidemiol 2021; 24: E210003.supl. https://doi.org/10.1590/1980549720210003.supl.2

24. Zeni ALB, Parisotto AV, Mattos G, Helena ETS. Utilização de plantas medicinais como remédio caseiro na Atenção Primária em Blumenau, Santa Catarina, Brasil / Use of medicinal plants as home remedies in Primary Health Care in Blumenau - State of Santa Catarina, Brazil. Ciênc. Saúde Colet. 2017; 22(8): 2703-2712. https://doi.org/10.1590/1413-81232017228.18892015

25. Trkulja V, Barić H. Current Research on Complementary and Alternative Medicine (CAM) in the Treatment of Anxiety Disorders: An Evidence-Based Review. Anxiety Disorders. 2020;1191:415- 
449. https://doi.org/10.1007/978-981-32-9705-0_22

26. Smith CA, Collins CT, Levett KM, Armour M, Dahlen HG, Tan AL, et al. Acupuncture or acupressure for pain management during labour. Cochrane Database Syst Rev.

2020;2(2):CD009232. https://doi.org/10.1002/14651858.CD009232.pub2

27. Neel K, Goldman R, Nothnagle M. Integrating doulas into hospital births: Provider perceptions of doulas and doula care [22C]. Obstetrics \& Gynecology. 2019; 133:37S. https://doi.org/10.1097/01.AOG.0000559443.76117.4a

28. Johnson A, Roberts L, Elkins G. Complementary and Alternative Medicine for Menopause. J Evid Based Integr Med. 2019;24:1-14. https://doi.org/10.1177/2515690X19829380

29. Macinko J, Mendonça CS. Estratégia Saúde da Família, um forte modelo de Atenção Primária à Saúde que traz resultados. Saúde em Debate. 2018; 42:18-37. https://doi.org/10.1590/0103$11042018 S 102$

30. Brasil, Ministério da Saúde. Relatório de Monitoramento Nacional das Práticas Integrativas e Complementares em Saúde nos Sistemas de Informação em Saúde. 2020 Disponível em: http://observapics.fiocruz.br/oferta-de-pics-cresce-na-atencao-primaria-e-especializada/ Acessado: $20 / 10 / 21$

31. Fjær EL, Landet ER , McNamara CL, Eikemo TA. The use of complementary and alternative medicine (CAM) in Europe. BMC Complement Med Ther. 2020;20(1):108. https://doi.org/10.1186/s12906-02002903-w

32. Nahin RL, Barnes PM, Stussman BJ. Insurance coverage for complementary health approaches among adult users: United States, 2002 and 2012. NCHS Data Brief, no 235. Hyattsville, MD: National Center for Health Statistics. 2016.

33. Barnes PM, Powell-Griner E, McFann K, Nahin RL. Complementary and alternative medicine use among adults: United States, 2002. Advance data from vital and health statistics; no 343. Hyattsville, MD: National Center for Health Statistics. 2004.

34. Cramer H. Meditation in Deutschland: Eine national repräsentative Umfrage. Complement Med Res. 2019;26(6):382-389. https://doi.org/10.1159/000499900

35. Ding D, Stamatakis E. Yoga practice in England 1997-2008: prevalence, temporal trends, and correlates of participation. BMC Res Notes. 2014; 7: 172 https://doi.org/10.1186/1756-0500-7-172

36. Macinko J, Upchurch DM. Factors Associated with the Use of Meditation, U.S. Adults 2017. J Altern Complement Med. 2019;25(9):920-927. https://doi.org/10.1089/acm.2019.0206

37. Saper RB, Eisenberg DM, Davis RB, Culpepper L,Phillips RS. Prevalence and patterns of adult yoga use in the United States: results of a national survey. Alt Ther Health Med. 2004;10(2):44-9.

38. Barros NF, Siegel P, Moura SM, Cavalari TA, Silva, LG, Furlanetti, M.R., Gonçalves, A.V. Yoga e promoção da saúde. Cien Saude Colet. 2014; 19(4):1305-1314. https://doi.org/10.1590/141381232014194.01732013

39. Goncalves JPB, Lucchetti G, Menezes PR, Vallada H. Complementary religious and spiritual interventions in physical health and quality of life: A systematic review of randomized controlled 
clinical trials. Plos One. 2017;12(10): e0186539. | https://doi.org/10.1371/journal.pone.0186539

40. Jazieh AR, Abuelgasim KA, Ardah HI, Alkaiyat M, Da'ar OB. The trends of complementary alternative medicine use among cancer patients. BMC Complement Med Ther. 2021;21(1):167. https://doi.org/10.1186/s12906-021-03338-7

41. Rao JK, Mihaliak K, Kroenke K, Bradley J, Tierney WM, Weinberger M. Use of complementary therapies for arthritis among patients of rheumatologists. Ann Intern Med. 1999;131(6):409-16. https://doi.org/10.7326/0003-4819-131-6-199909210-00003

42. Kaboli PJ, Doebbling BN, Saag KG, Rosenthal GE. Use of complementary and alternative medicine by older adults with arthritis: a population-based study. Arthritis Rheum 2001;45:398-403. https://doi.org/10.1002/1529-0131(200108)45:4<398::AID-ART354>3.0.C0;2-I

43. Quandt SA, Chen, H, Grzywacz JG, Grzywacz JG, Bell RA, Lang W, Arcury TA. Use of complementary and alternative medicine by persons with arthritis: results of the National Health Interview Survey. Arthritis Rheum 2005;53 (5):748-755. https://doi.org/10.1002/art.21443

44. Quandt SA, Verhoef MJ, Arcury TA, Lewith GT, Steinsbekk A, Kristoffersen AE, et al. Development of an international questionnaire to measure use of complementary and alternative medicine (ICAM-Q). J Altern Complement Med. 2009; 15(4): 331-9. https://doi.org/10.1089/acm.2008.0521

45. Arcury TA, Bell RA, Snively BM, Smith SL, Skelly AH, Wetmore LK, Quandt SA. J Complementary and alternative medicine use as health self-management: rural older adults with diabetes. Gerontol B Psychol Sci Soc Sci. 2006;61(2):S62-70. https://doi.org/10.1093/geronb/61.2.s62 